\title{
ФАКТОРЫ РИСКА В РАЗВИТИИ ДЕМИНЕРАЛИЗАЦИИ КОСТНОЙ ТКАНИ У НЕДОНОШЕННЫХ НОВОРОЖДЕННЫХ
}

\author{
Н.Г. Киселева, Т.Е. Таранушенко \\ ФГБОУ ВО «Красноярский государственный медицинский университет \\ им. проф. В.Ф. Войно-Ясенецкого» МЗ, г. Красноярск
}

Пристальное внимание к проблеме остеопении обусловлено значительной распространённостью данной патологии, а также необходимостью оптимизации профилактических и лечебных мероприятий нарушений фосфорно-кальциевого обмена у недоношенных. Основные причины развития остеопении известны, вместе с тем, продолжают активно изучаться факторы риска и биохимические маркёры нарушений метаболизма в костной ткани у новорожденных с разным сроком гестации.

Одним из основных индикаторов низкой минерализации скелета и нарушения процессов ремоделирования кости у недоношенных, является щелочная фосфатаза, представленная у младенцев в основном костной изоформой (90\% от показателя общей щелочной фосфатазы), что позволяет рассматривать данный фермент как скрининговый тест доступный в рутинной практике неонатолога.

ЦЕЛЬ НАСТОЯЩЕГО ИССЛЕДОВАНИЯ - ПроаНалИЗИровать факторы рИска остеопенИи У недоношенных новорожденных.

МАТЕРИАЛЫ И МЕТОДЫ ИССЛЕДОВАНИЯ. ВЫПоЛнено ретроспектИвное одноцентровое описательное исследование, включающее анализ 85 историй болезни недоношенных новорожденных с очень низкой и экстремально низкой массой тела (ОНМТ и ЭНМТ), госпитализированных в отделение патологии новорожденных и недоношенных детей КГБУЗ «КККЦОМД». Критериями включения для основной (целевой) группы были увеличенные значения сывороточной щелочной фосфатазы (в отсутствии данных за холестаз и патологию обмена билирубина), позволившие сформировать группу из 45 недоношенных (медиана гестационного возраста - 27 нед [min - 24, max - 32]). Группу контроля составили 40 новорожденных с нормальными показателями щелочной фосфатазы, сопоставимые по возрасту (медиана гестационного возраста - 27 нед [min - 25, max - 32]), гендерному признаку, показателям физического развития (оценка по диаграмме Фентона) и по клиническому статусу. Средний возраст женщин на момент настоящей беременности также не имел значимых различий, медиана возраста в 1 группе составила 32 года [min - 17; max - 42], во второй группе - 30 лет [min - 19; $\max -39$ ].

Статистическая обработка материала выполнена с использованием персонального компьютера и прикладных программ MS Excel 2016 (Microsoft Corporation, CША), Statistica 8.0 (StatSoft Inc., США). Статистическая значимость различий полученных данных определялась с помощью критериев хи-квадрат, Уилкоксона, Манна-Уитни. Критическое значение уровня значимости принималось равным $5 \%(p<0,05)$.

РЕЗУЛЬТАТЫ ИССЛЕДОВАНИЯ. На первом этапе исследования оценивались пренатальные факторы риска развития остеопении. Известно, что важным условием адекватного внутриутробного транспорта кальция к плоду является благоприятное течение беременности и нормальное функционирование плаценты. Учитывая связь акушерского анамнеза матери с последующим нарушением фосфорно-кальциевого обмена у новорожденных, нами изучены основные факторы, предрасполагающие к развитию рассматриваемой патологии: наличие плацентарных нарушений, преэклапсия, сахарный диабет, курение, ЗВУР плода. Установлено, что указания на патологию плаценты отмечались в обеих группах, при этом хроническая фетоплацентарная недостаточность регистрировалась чаще в первой группе - 23 беременных (51\%) против 14 женщин (35\%) контрольной группы $(p<0,05)$. Частота хориоамнионита в рассматриваемых группах не имела достоверных различий и составила 4,5-7\%. Преэклампсия и табакококурение регистрировалось с примерно одинаковой частотой 22-27\% случаев в обеих группах. 


\section{СБОРНИК ТЕЗИСОВ}

XVII Российская научно-практическая конференция детских эндокринологов «Достижения науки в практику детского эндокринолога»

На втором этапе исследования оценивались постнатальные факторы, предрасполагающие к развитию остеопении: низкая масса тела, ИВЛ, получение медикаментов, нарушающих метаболизм кальция, БЛД, факт пролонгированного парентерального питания.

При оценке состояния новорожденного задержка внутриутробного развития плода по гипопластическому типу выявлена у 15 недоношенных новорожденных (33\%) первой группы и у 9 младенцев (22\%) второй группы. Медиана массы тела при рождении у недоношенных первой группы составила 850 г. [min - 540; max - 1200], во 2 группе - 950 г. [min - 780; $\max$ - 1190].

По данным исследования респираторную поддержку получали 42 младенца (93\%) из целевой группы и 35 (87,5\%) пациентов из группы контроля. Важно, что продолжительность АИВЛ была в 2,8 раза дольше у новорожденных первой группы и составила в среднем 11 дней против 4 дней во второй группе.

Результаты нашего исследования показали более высокую частоту случаев бронхолегочной дисплазии (БЛД) у недоношенных 1 группы чаще - 77\% против 52\% недоношенных из второй группы $(p<0,05)$. По мнению ряда авторов на фоне формирования БлД у недоношенных может увеличиваться частота остеопений бронхолёгочной дисплазией, при этом триггером костных нарушений является повышенная продукция провоспалительных цитокинов с последующей активацией остеокластов.

Известно, что ГКС оказывают существенное влияние на структуру костной ткани через угнетение секреции ИФР-1 и СТГ, снижение кишечной абсорбции и почечной реабсорбции кальция, а также нарушение функции остеобластов. Указанные обстоятельства приводят к преобладанию процессов костной резорбции над остеомоделированием и изменению микроархитектоники кости.

По нашим данным младенцы 1 группы чаще получали терапию системными и ингаляционными ГКС - 32 пациента (71\%) по сравнению с 13 недоношенным (32\%) контрольной группы. Продолжительность введения системных ГКС в сравниваемых группах сопоставима и составила $7 \pm 1$ дней. Терапия ингаляционными ГКС продолжалась $10 \pm 1$ дней в контрольной группе и была более пролонгированной у недоношенных с остеопенией $-23 \pm 1$ день $(p<0,05)$.

По данным литературы метилксантины и мочегонные препараты снижают реабсорбцию электролитов в почечных канальцах. Метилксантины использовались в обеих группах в $100 \%$ случаев. Вместе с тем, указанная терапия у новорожденных 1 группы отличалась большей продолжительностью. Так, медиана длительности лечения кофеином в 1 группе составила 40 дней, во второй группе - 29 дней. Доля детей, потребовавших терапию диуретиками, составила 24\% в 1 группе, против 17\% в группе контроля, с медианой продолжительности лечения 31 день и 17 дней соответственно.

Важную роль в развитии метаболических нарушений костной ткани имеет питание. В настоящем исследовании рассмотрен возможный вклад пролонгированного парентерального питания, при котором имеется недостаточное поступление (допустимый порог внутривенной дотации) и сниженное усвоение кальция и фосфора, в снижение минерализации костной ткани. Результаты исследования показали более длительное полное и частичное парентеральное питания у младенцев в 1 группе по отношению к группе контроля, в среднем на 5-6 дней, что может быть причиной последующего нарушения метаболизм в костной ткани и повышением щелочной фосфатазы у недоношенных.

На третьем этапе исследования изучены и сопоставлены клинико-лабораторные особенности пациентов в изучаемых группах.

По данным литературы, остеопения у недоношенных протекает в большинстве случаев бессимптомно. Основным диагностическим критерием метаболической болезни костей является быстрое нарастание в динамике и значительно повышенный уровень общей щелочной фосфатазы, что обусловлено преобладанием у младенцев костной изоформы. Результаты проведённого исследования показали, что уровень щелочной фосфатазы характеризовался устойчивым повышением, который регистрировался в течение всего периода наблюдения 
в нескольких лабораторных образцах, медиана показателя составила - 943 Ед/л [min - 600; $\max -1784]$.

Другим скрининговым маркером нарушенного метаболизма костной ткани, является фосфор. По мнению ряда авторов, уровень сывороточного фосфата менее 2 ммоль/л отражает риск остеопении, а концентрация менее 1.8 ммоль/л требует проведения диагностической рентгенографии костей. По нашим данным концентрация фосфора крови соответствовала лабораторной норме, но была ниже референсных значений у $75 \%$ обследованных, при этом у $25 \%$ пациентов показатель оказался ниже 2.0 ммоль/л, а у 50\% детей уровень фосфора был менее 1.8 ммоль/л, что указывает на высокий риск развития метаболической болезни костей.

Уровень кальция при остеопении обычно остается в норме или незначительно повышается, что обусловлено компенсаторным увеличением секреции ПТГ паращитовидными железами и мобилизацией кальция из костного депо в кровь. Содержание общего и ионизированного кальция в крови у всех недоношенных соответствовало физиологическим возрастным значениям.

У трёх пациентов при проведении планового рентгенологического исследования описаны признаки остеопороза проксимального отдела метафиза плечевых костей. Данные изменения костной ткани при стандартной рентгенографии свидетельствуют о существенном снижении костной массы (на 30-35\%) и позволяют обсуждать значительное нарушение минеральной плотности костей у данных новорожденных.

Клинические проявления остеопении в виде перелома левой лучевой кости зарегистрировано только у одного пациента, при этом уровень 25(OH)D3 у этого младенца соответствовал норме и составил 76 нг/мл. Данные особенности подтверждают концепцию формирования механизмов гидроксилирования витамина D у плода к 24 нед внутриутробного развития и возможности самостоятельного синтеза кальцидиола при адекватном поступлении материнского холекальциферола.

\section{Выводы.}

1. К наиболее вероятным анамнестическим факторам риска развития остеопении недоношенных следует отнести признаки антенатального неблагополучия (хроническая фетоплацентарная недостаточность, сахарный диабет инсулинозависимый), а также сочетание недоношенности с задержкой внутриутробного развития по гипопластическому типу.

2. Постнатальными факторами, способствующими повышению риска остеопении, можно считать пролонгированное применение ИВЛ, прием ГКС, диуретиков и метилксантинов.

3. Повышение щелочной фосфатазы у недоношенных новорожденных характеризуется устойчиво высокими значениями в сочетании со снижением уровня фосфора при нормальных значениях кальция в сыворотке крови.

4. Клинические проявления остеопении выявлены у 1 новорожденного (перелом лучевой кости) при нормальных показателях кальция и фосфора и адекватной обеспеченности витамином D3. 